\title{
Vulnerability in high-functioning persons aged 65 to 70 years: the importance of the fear factor
}

\author{
Laurence Seematter-Bagnoud ${ }^{1,2}$, Brigitte Santos-Eggimann ${ }^{2}$, Stéphane Rochat ${ }^{1}$, Estelle Martin ${ }^{1}$, \\ Athanassia Karmaniola ${ }^{2}$, Kamiar Aminian ${ }^{3}$, Chantal Piot-Ziegler ${ }^{4}$ and Christophe J. Büla ${ }^{1}$ \\ ${ }^{1}$ Service of Geriatric Medicine and Geriatric Rehabilitation, University of Lausanne Hospital Center, \\ ${ }^{2}$ Institute of Social and Preventive Medicine, University of Lausanne Hospital Center, ${ }^{3}$ Laboratory of \\ Movement Analysis and Measurements (LMAM), Ecole Polytechnique Federale de Lausanne (EPFL), \\ ${ }^{4}$ Institute of Psychology, Social and Political Sciences Faculty, University of Lausanne, Switzerland
}

ABSTRACT. Background and aims: Falls efficacy has been shown to predict functional decline, but whether it is independently associated with frailty is still unclear. This study investigated the cross-sectional association between falls efficacy and the frailty phenotype in high-functioning older persons. Methods: Subjects $(n=861)$ were a sub-sample of community-dwelling persons aged 65 to 70 years enrolled in the "Lc65+" cohort, who had gait assessment. Data included demographics, functional, cognitive, affective and health status, as well as measures of physical performance. Falls efficacy was measured with the Falls Efficacy Scale-International (FES-I) and frailty with Fried's criteria. Participants were categorized into robust (no frailty criterion) and vulnerable (1 or more criteria). Low falls efficacy was defined as a FES-I score in the lowest quartile. Results: Overall, $23.9 \%$ of participants were vulnerable. Compared with robust participants, they were more likely to report low falls efficacy $(43.3 \%$ vs $19.1 \%, \mathrm{p}<0.001)$ and had poorer health and functional and mental status. They had slower gait speed $(1.07 \pm 0.18$ vs $1.15 \pm 0.15 \mathrm{~m} / \mathrm{s}, \mathrm{p}<0.001)$ and increased gait speed variability (coefficient of variation $4.10 \pm 4.03$ vs $3.33 \pm 1.45 \%, p<0.001$ ), although only 6 participants $(0.7 \%)$ fulfilled Fried's slow walking criterion. In multivariate analysis, low falls efficacy remained associated with being vulnerable (adjusted $O R$ 1.80, 95\% CI 1.19-2.74, $\mathrm{p}=0.006$ ), independent of comorbidity, functional status, falls history and gait performance. Conclusion: In high-functioning older persons, low falls efficacy was associated with vulnerability, even after controlling for gait performance and falls history. Whether low falls efficacy is a potential target on the pathway leading to frailty should be further examined prospectively.

(Aging Clin Exp Res 2010; 22: 212-218)

${ }^{\circ}$ 2010, Editrice Kurtis

\section{INTRODUCTION}

Fear of falling is a frequent problem in older persons, affecting as many as 20-60\% of those aged over 65 years who live in the community $(1,2)$. It may be assessed directly or through "falls self-efficacy". As proposed by Bandura, self-efficacy represents the degree of confidence a person has in his/her ability to perform a specific activity, and influences behaviours regarding that activity (3). In the present context, fear of falling might be defined as "low perceived efficacy at avoiding falls during essential, nonhazardous activities of daily living (4). Fear of falling may thus lead to restriction in these activities, initiating a vicious cycle of decline in physical abilities (5). Fear of falling has also been associated with future functional decline in high-functioning older women even in the absence of activity restriction (6).

Frailty also places older people at higher risk for falls and disability, as well as hospitalizations, institutionalisation and death $(7,8)$. Among indexes developed to characterize frailty, the definition of Fried's et al. (7) is the most widely used (9), and relies on assessment in five domains: nutrition/sarcopenia (involuntary weight loss), endurance (fatigue), physical inactivity, muscle strength (low grip strength) and mobility (slow walking). Frailty is defined along a continuum: individuals meeting one or two criteria are classified as pre-frail, and those with three or more criteria are classified as frail. Both frail and pre-frail individuals have been shown to experience more frequent adverse

Key words: Fear of falling, frailty, gait, vulnerability.

Correspondence: Dr. Laurence Seematter-Bagnoud, Service de Gériatrie et Réadaptation Gériatrique, Centre Universitaire de Traitement et de Réadaptation, Chemin de Sylvana 10, CH-1066 Epalinges, Switzerland.

E-mail: Laurence.Seematter-Bagnoud@chuv.ch

Received February 17, 2009; accepted in revised form May 5, 2009.

First published ahead of print November 27, 2009 as DOI: 10.3275/6705. 
outcomes and may thus be considered as vulnerable (7-9).

Previous studies have found an association between fear of falling and slow gait speed (10-12) and therefore one might also expect fear of falling to be associated with frailty. Surprisingly, this relationship has not been well studied $(11,13)$. In particular, it still remains to be verified whether it is truly independent of or is mediated by gait performance. Clarifying this issue is important, in order to determine whether fear of falling is a risk factor for frailty and a possible specific target for interventions aiming at disability prevention.

The aim of this study was to determine whether fear of falling, as measured by low falls efficacy, is associated with early signs of impending frailty in community-dwelling older persons aged 65 to 70 , independent of gait performance and previous falls history.

\section{METHODS}

\section{Study design and population}

Participants were a subsample of subjects enrolled in the Lausanne cohort 65+ (Lc65+) on age-related frailty in community-dwelling young-old persons. The Lc65+ recruitment and methods have been described in detail elsewhere (14). Briefly, a random sample of 3056 persons born between 1934 and 1938 residing in Lausanne, Switzerland, was contacted. From the 1310 subjects who completed baseline data collection (Fig. 1), 861 had gait parameters recorded using body-fixed sensors (15). The main reasons for missing gait parameters were unavailability of the recording device (24.9\%), inability to walk due to health and/or safety problems (5.3\%), and refusal (1.3\%). Participants who underwent gait recording were more frequently men, were married, had higher education and better functional status, and reported fewer falls (data not presented) than those who did not.

\section{Data collection}

Information about socio-economic status, education, self-rated health, chronic diseases (out of a list of 12 chronic diseases), physical activity, previous falls in preceding 12 months, and depressive symptoms, as well as difficulties or help in basic and instrumental activities of daily living (ADL) was collected through a postal questionnaire.

Additional data were collected during an in-person visit, including anthropometrics, and measures of cognition, grip strength, and gait parameters (14). Data collection was performed by trained research assistants using a standardized protocol. Maximal grip strength in the right hand (best performance of 3 trials) was measured on a BASELINE ${ }^{\circledR}$ hand-held dynamometer.

Gait assessment: Gait speed $(\mathrm{m} / \mathrm{s})$ was measured over 20 meters in a well-lighted walkway with participants walking at self-selected speed, by the Physilog ${ }^{\circledR}$ system

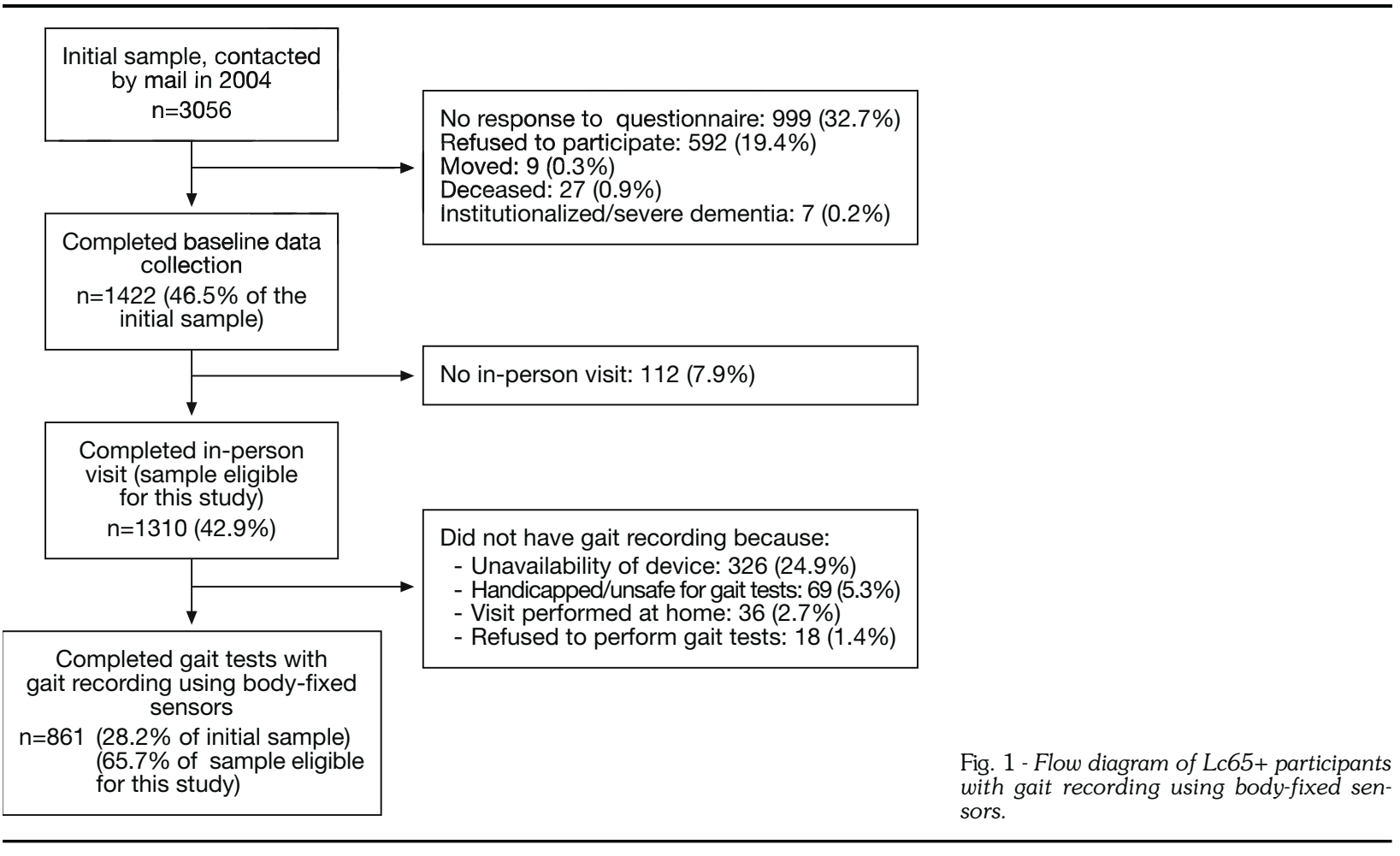

213 Aging Clin Exp Res, Vol. 22, No. 3 
[BioAGM, Tour-de-Peilz, Switzerland (15)]. Gait speed variability was assessed with the coefficient of variation $(\mathrm{CV}$ in \%) defined as the standard deviation divided by the mean value of gait speed for each stride (16).

\section{Falls efficacy assessment}

Falls efficacy was measured with the Falls-EfficacyScale International (FES-I (17)) which assesses a person's concern about falling while performing 16 activities of daily living (e.g., taking a bath, answering the phone, etc.). Four answers are possible, ranging from "very concerned" to "not at all concerned" with total scores ranging from 16 to 64 . As higher scores indicate lower falls efficacy, FES-I scoring was subsequently reversed and standardized (rsFES-I) for this analysis, to provide scores ranging from 0 to 100 , higher scores indicating higher falls efficacy.

In the main analysis, falls efficacy was dichotomized as low vs normal, where low falls efficacy was defined as a score in the lowest quartile at the rsFES-I. In secondary analyses, the rsFES-I score was also used as a continuous variable.

\section{Frailty assessment and definition}

Frailty was measured according to the following criteria (7):

- Low muscle strength: cut-off for low grip strength as used by Fried et al. (7);

- Poor nutrition: self-reported unintentional weight loss during preceding 12 months;

- Poor endurance: self-reported lack of energy and fatigue during preceding 4 weeks;

- Slow walking: cut-off for slow gait speed as used by Fried et al. (7);

- Low physical activity: defined as doing less than 20 minutes of sports per week, or walking less than 90 minutes per week. Participants fulfilling these criteria were nevertheless considered active if they reported a high amount of daily usual physical activity such as walking stairs, or lifting weights.

Based on these five criteria, three categories were defined: robust (0 criterion), pre-frail (1-2 criteria), and frail (3+criteria). Data on frailty criteria were available for all except 15 participants, for whom information about physical activity was missing. We considered these participants as not fulfilling the frailty physical activity criterion, allowing their classification in robust $(n=7)$ and pre-frail $(n=8)$ categories. Given the very low proportion of frail subjects $(0.8 \%)$, the frailty variable was dichotomized into robust $(0$ criterion) and vulnerable (one or more criteria) in subsequent analyses.

\section{Statistical analyses}

Characteristics of robust and vulnerable participants were compared with Pearson's chi-squared test for cate- gorical variables and Student's $t$-test for continuous variables.

The prevalence of low falls efficacy was compared between robust and vulnerable subjects after categorization of the sample into non-fallers, previous single fallers, and multiple fallers, with chi-squared tests and tests for trend. Gait speed was compared within robust and vulnerable participants after stratification for falls efficacy status, with Student's $t$-test.

Multivariate logistic regression analyses were performed to examine the association between low falls efficacy (dichotomous) and frailty status. Adjustment variables were chosen on the basis of their likely relationships with frailty and fear of falling and included: age, gender, living alone, education, falls in the previous year, cognitive impairment, depressive symptoms, comorbidity, and functional impairment (Model 1). Gait parameters were secondarily included (Model 2).

Sensitivity analyses were performed after exclusion of: a) participants $(n=15)$ with missing data on one frailty criterion; b) participants $(n=7)$ with 3 or more frailty criteria; c) participants $(n=6)$ who had slow gait speed according to Fried's criterion. Lastly, additional analyses were also performed with the rsFES-I score as a continuous variable. Analyses were processed by Stata, version 10.0.

The study was approved by the institution review board, and written consent was obtained from all participants during the in-person visit.

\section{RESULTS}

Overall, $75.3 \%$ (648/861) of subjects did not fulfill any frailty criteria (robust group). In contrast, $24.7 \%$ (213/861) were considered as vulnerable in this analysis, because they had one $(20.2 \%)$, two $(3.7 \%)$, three $(0.7 \%)$ or four $(0.1 \%)$ frailty criteria. None of the participants met all five criteria. Among vulnerable subjects, low grip strength was observed in almost half $(101 / 213,47.4 \%)$, involuntary weight loss in one-third $(72 / 213,33.8 \%)$ and low physical activity $(42 / 205,19.7 \%)$ and exhaustion $(39 / 213,18.3 \%)$ in one-fifth. Slow gait speed was observed in only $6(2.8 \%)$ of the 213 vulnerable participants.

The characteristics of the population and comparisons between robust and vulnerable participants are listed in Table 1. The latter participants were slightly older, more likely to live alone and to report comorbidity and depressive symptoms. The proportion of participants with cognitive impairment tended to be higher among those vulnerable (3.4\% vs $1.6 \%)$. Vulnerable participants were also more likely to report functional impairment and falls in the previous year, and to have low falls efficacy. Lastly, vulnerable participants had slower gait speed and increased gait speed variability.

Figure 2 displays the prevalence of low falls efficacy in robust and vulnerable participants stratified by fall frequency, and shows a double gradient in this association. 
Table 1 - Baseline characteristics of study population and comparisons between robust and vulnerable participants.

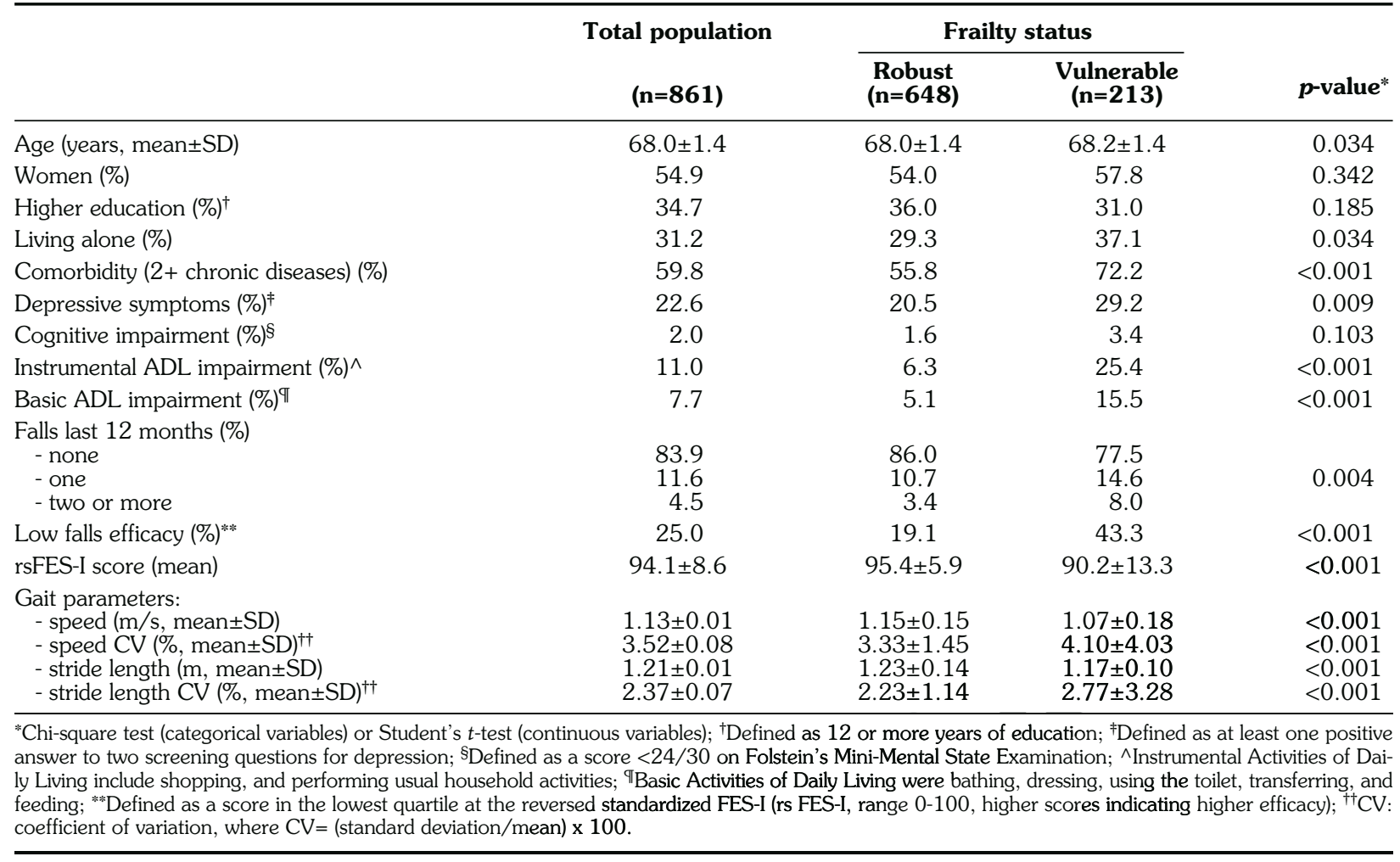

First, within each frailty category, the prevalence of low falls efficacy increased gradually according to falls frequency, with statistically significant trends in both robust and vulnerable groups (both $p<0.001$ ). Second, within each falls category, the prevalence of low falls efficacy was higher in vulnerable than in robust participants.

Similarly (Fig. 3), mean gait speed was significantly slower in participants with low falls efficacy compared with the others, among both robust $(1.16 \pm 0.15$ vs $1.09 \pm 0.15$ $\mathrm{m} / \mathrm{s}, p<0.001)$ and vulnerable $(1.11 \pm 0.16$ vs $1.05 \pm 0.17$ $\mathrm{m} / \mathrm{s}, p=0.024$ ) groups.

Table 2 lists results from bivariate and multivariate analyses predicting prevalent vulnerability. In the bivariate analysis, subjects with low falls efficacy had three times higher odds of being vulnerable. After adjustment for age, gender, education, living alone, cognitive and affective status, history of falls, comorbidity and functional status, low falls efficacy remained significantly associated with higher odds (Model 1: adjOR 2.03, 95\% CI 1.35-3.05, $p=0.001$ ) of being vulnerable. Further adjustment for gait performance (Model 2) only marginally modified this association (adjOR 1.80, 95\% CI 1.19-2.74, $p=0.006$ ). Beside low falls efficacy, gait speed and gait speed variability were also independently associated with being vulnerable, together with reporting comorbidity and impairment in instrumental ADLs. Specifically, for a decrease in gait speed of $0.1 \mathrm{~m} / \mathrm{s}$, the odds of being vulnerable increased by $8 \%$.

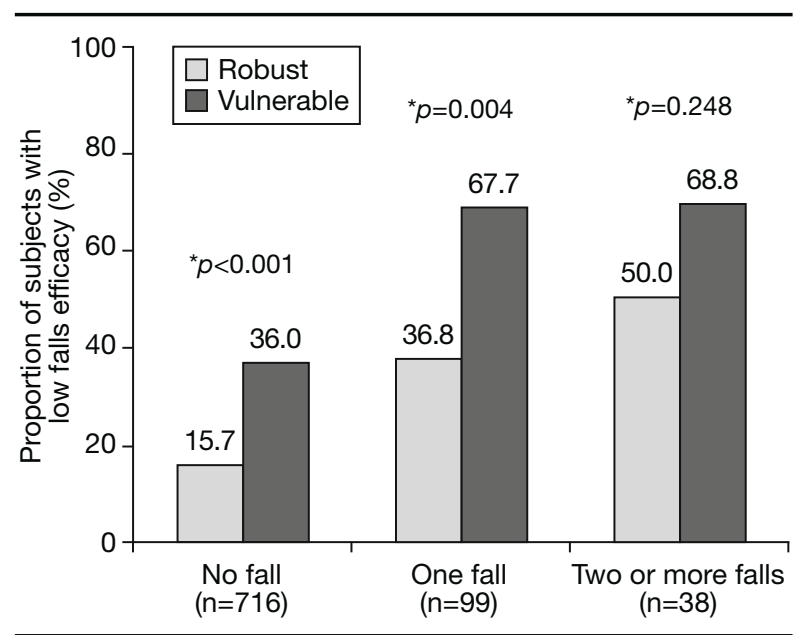

Fig. 2 - Prevalence of low falls efficacy according to frailty status among non-fallers, single fallers, and multiple fallers. "p-value from chi-square test comparing robust and vulnerable participants. 


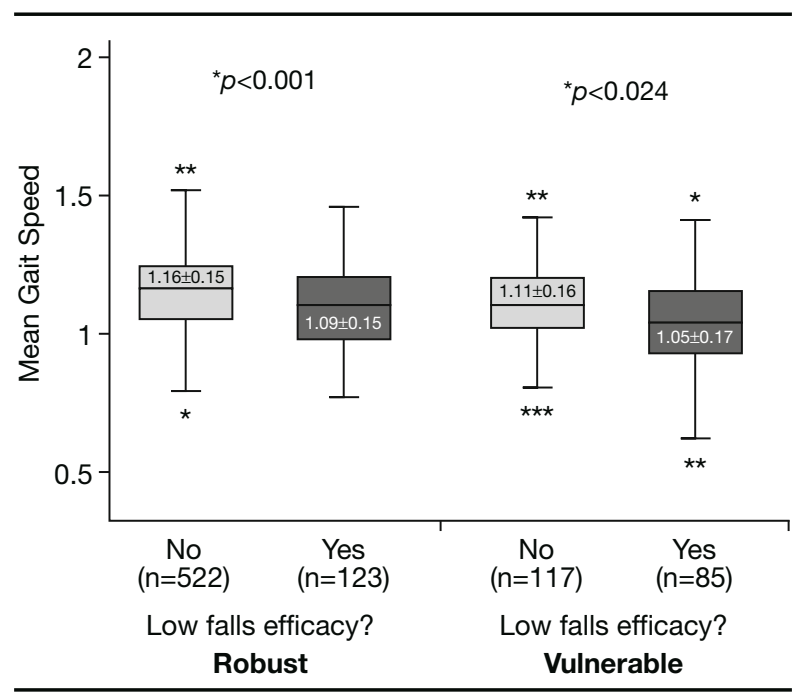

Fig. 3 - Gait speed in robust and vulnerable participants, according to falls efficacy status. "p-value from Student's t-test, comparing mean gait speed in confident and fearful participants among robust and vulnerable groups.

In the additional analysis with the rsFES-I score as a continuous variable in the same multivariate model, falls efficacy remained independently associated with vulnerability. A one-point increase in falls efficacy at rsFES-I decreased the odds of being vulnerable by $3 \%$ (adjOR 0.97, 95\% CI 0.94-0.99, $p=0.011$ ). Lastly, all three sensitivity analyses (i.e., excluding participants with missing data on one frailty criterion, those with 3 or more frailty criteria, and those with slow gait speed according to Fried's criterion) led to similar results.

\section{DISCUSSION}

In these high-functioning persons aged 65 to 70 years, low falls efficacy was associated with prevalent vulnerability, independent of gait performance and falls history. This relationship remained even when excluding the $0.7 \%$ of the sample who met Fried's criterion of slow walking speed (7). To our knowledge, this is the first study to document this independent relationship between a measure of fear of falling and early markers of vulnerability in relatively young, well-functioning older persons.

This result is important from several perspectives. First, it suggests that low falls efficacy may be a specific step and a target for intervention in the pathway leading to frailty and disability, independent of mobility impairment and falls. Second, the persistence of the association even after controlling for gait performance provides new insight into the type of intervention that may be indicated for frailty and disability prevention. Indeed, this observation may explain negative results observed in some studies based only on gait training (18) and suggests that interventions specifically targeting fear of falling may help to achieve improved results in frailty prevention (19). The observed link between falls efficacy and vulnerability also strengthens the importance of psychological factors as well as biological and social factors, as components of frailty.

Overall, these results encourage further investigation of the possible independent contribution of fear of falling in

Table 2 - Results from bivariate and multivariate analyses of the association between low falls efficacy and vulnerability.

\begin{tabular}{|c|c|c|c|c|c|c|c|c|c|}
\hline \multirow[b]{2}{*}{ Characteristics } & \multicolumn{3}{|c|}{ Bivariate results } & \multicolumn{3}{|c|}{ Adjusted results, Model 1* } & \multicolumn{3}{|c|}{ Adjusted results, Model 2** } \\
\hline & Odds Ratio & $95 \% \mathrm{CI}$ & $p$-value & Odds Ratio & $95 \% \mathrm{CI}$ & p-value & Odds Ratio & $95 \% \mathrm{CI}$ & $p$-value \\
\hline Low falls efficacy ${ }^{\dagger}$ & 3.24 & $2.29-4.58$ & $<0.001$ & 2.03 & $1.35-3.05$ & 0.001 & 1.80 & $1.19-2.74$ & 0.006 \\
\hline Comorbidity (2+ chronic diseases) & 2.05 & $1.46-2.89$ & $<0.001$ & 1.65 & $1.15-2.39$ & 0.007 & 1.65 & $1.14-2.40$ & 0.009 \\
\hline Instrumental ADL impairment ${ }^{\S}$ & 5.02 & $3.17-7.95$ & $<0.001$ & 3.06 & $1.69-5.55$ & $<0.001$ & 2.87 & $1.56-5.27$ & 0.001 \\
\hline Gait speed (m/s) & 0.05 & $0.02-0.14$ & $<0.001$ & - & - & - & 0.20 & $0.06-0.67$ & 0.009 \\
\hline Speed variability $(\%)^{\wedge}$ & 1.14 & $1.07-1.22$ & 0.001 & - & - & - & 1.10 & $0.99-1.22$ & ns \\
\hline Age & 1.13 & $1.01-1.26$ & 0.035 & 1.13 & $1.00-1.28$ & 0.047 & & & ns \\
\hline Female gender & 1.16 & $0.85-1.59$ & ns & & & ns & & & ns \\
\hline Higher education & 0.80 & $0.57-1.11$ & ns & & & ns & & & ns \\
\hline Living alone & 1.42 & $1.02-1.97$ & 0.033 & & & ns & & & ns \\
\hline Depressive symptoms & 1.60 & $1.12-2.29$ & 0.009 & & & ns & & & ns \\
\hline Cognitive impairment & 2.21 & $0.83-5.90$ & ns & & & ns & & & ns \\
\hline Basic ADL impairment & 3.42 & $2.03-5.73$ & $<0.001$ & & & ns & & & ns \\
\hline Falls last 12 months & 1.78 & $1.20-2.64$ & 0.004 & & & ns & & & $\mathrm{ns}$ \\
\hline
\end{tabular}


the causal pathway leading to frailty and disability (5).

Another original finding of this study is the double gradient shown in the association between falls, fear of falling, and frailty. The prevalence of low falls efficacy increased steadily as falls frequency increased but, within each falls category, low falls efficacy consistently remained more prevalent in vulnerable than in robust participants. Although the relationships between falls, poor falls efficacy, and frailty have been shown separately in previous studies $(1,6,8,9,20)$, current results provide new insight into their complex interplay. In particular, they suggest the mediating role of falls in the relationship between low falls efficacy and frailty. Although this cross-sectional analysis precludes drawing any conclusion about the chronology of falls, fear of falling and frailty occurrence, the observation of a double gradient supports the hypothesis that interventions directed at improving falls efficacy may also play a significant role in postponing frailty and disability.

Results from this study also add to previous evidence about the potential role of gait speed as a prognostic marker in older persons. Even among these well-functioning older persons, who essentially did not meet the usual frailty cut-off for slow walking, gait speed and its variability distinguished persons with different vulnerability status. These differences all exceeded the $0.05 \mathrm{~m} / \mathrm{s}$ threshold considered as clinically meaningful (20), and future work should determine whether these subtle differences are predictive of adverse outcomes.

This study has several limitations. First, gait performance was recorded only in a subsample of the cohort. Although the main reason for missing data (i.e., unavailability of gait measuring device) occurred randomly, about $8.1 \%$ of participants were excluded because they could not perform the gait tests. As expected, these participants were in worse health than those who did perform the tests. Despite this selection bias, the observation of the independent association between poor falls efficacy and vulnerability among the fittest participants further emphasizes the interest of this finding from a preventive perspective. Second, the use of imputation on missing frailty criteria may have resulted in misclassification of some participants, underestimating their frailty status. However, this imputation process concerned only 15 individuals (1.7\%), and sensitivity analyses restricted to individuals with complete frailty data provided similar results. Third, the vulnerable group was heterogeneous, as it included both prefrail and frail individuals. Very few $(0.8 \%)$ participants were frail, precluding examination of a possible dose-response effect across the three frailty groups. Therefore, findings essentially apply to pre-frail individuals. Lastly, as previously mentioned, the cross-sectional design of the study prevents any conclusion about the chronology and the causality of the observed relationships between fear of falling, vulnerability, and falls history. Longitudinal studies are needed to further investigate the possible reciprocal relationship between fear of falling and frailty, most probably through a vicious cycle of reduced physical activity, increased fear of falling, and increased frailty.

This study has several strengths, which include the large number of participants in a narrow age range, making it a homogeneous cohort of well-functioning individuals. A unique feature of the current study was also the detailed assessment of gait speed and variability, which was recorded over a longer distance than usual. Lastly, an extensive set of potential confounders, including performance-based measures, was collected and adjusted for in the analyses.

In conclusion, this work shows the existence of a relationship between fear of falling and frailty, and suggests that it is potentially mediated by falls history, but remains - at least partially - independent of gait performance. These findings corroborate our initial hypothesis that fear of falling may be an important component of the transition to frailty. They also lend preliminary support to targeting falls efficacy in the pathway leading to frailty and disability, but longitudinal studies are needed to confirm these results and decide how best to intervene.

\section{ACKNOWLEDGEMENTS}

We would like to thank all participants in the Lc65+ study, together with research assistants: M. Rimaz, V. Chabloz, C. Borer, M-M. Mathey and M. Thomi, as well as M. Jacot-Guillarmod for secretarial assistance, and J.C. Beck for comments on the manuscript. We are grateful to the Loterie Romande (non-profit organization supporting research and social projects) for their grant to the Foundation Lausanne Cohort Lc65+. This study was also supported by grants from the Swiss National Scientific Foundation (325200-109401, Dr. C. Büla) and the Leenaards Foundation (Dr. S. Rochat). Prof. B. Santos-Eggimann is the principal investigator in the Lc65+ cohort. Dr. L. Seematter-Bagnoud had full access to all the data in the study and takes responsibility for data integrity and the accuracy of data analysis.

\section{REFERENCES}

1. Delbaere K, Crombez G, Vanderstraeten G, Willems T, Cambier D. Fear-related avoidance of activities, falls and physical frailty. A prospective community-based cohort study. Age Ageing 2004; 33: 368-73.

2. Zijlstra GA, van Haastregt JC, van Eijk JT, van RE, Stalenhoef PA, Kempen GI. Prevalence and correlates of fear of falling, and associated avoidance of activity in the general population of community-living older people. Age Ageing 2007; 36: 304-9.

3. Bandura A. Self-efficacy mechanism in human agency. Am Psychol 1982; 37: 122-47.

4. Tinetti ME, Richman D, Powell L. Falls efficacy as a measure of fear of falling. J Gerontol 1990; 45: 239-43.

5. Deshpande N, Metter EJ, Lauretani F, Bandinelli S, Guralnik J, Ferrucci L. Activity restriction induced by fear of falling and objective and subjective measures of physical function: a prospective cohort study. J Am Geriatr Soc 2008; 56: 615-20.

6. Carriere I, Colvez A, Favier F, Jeandel C, Blain H. Hierarchical components of physical frailty predicted incidence of dependency in a cohort of elderly women. J Clin Epidemiol 2005; 58: 1180-7.

7. Fried LP, Tangen CM, Walston J et al. Frailty in older adults: ev- 
idence for a phenotype. J Gerontol A Biol Sci Med Sci 2001; 56 : M146-56.

8. Woods NF, LaCroix AZ, Gray SL et al. Frailty: emergence and consequences in women aged 65 and older in the Women's Health Initiative Observational Study. J Am Geriatr Soc 2005; 53: 1321-30.

9. Bandeen-Roche K, Xue QL, Ferrucci L et al. Phenotype of frailty: characterization in the women's health and aging studies. J Gerontol A Biol Sci Med Sci 2006; 61: 262-6.

10. Maki BE. Gait changes in older adults: predictors of falls or indicators of fear? J Am Geriatr Soc 1997; 45: 313-20.

11. Kressig RW, Gregor RJ, Oliver A et al. Temporal and spatial features of gait in older adults transitioning to frailty. Gait Posture 2004; 1: 30-5.

12. Rochat S, Martin E, Piot-Ziegler C, Najafi B, Aminian K, Bula CJ. Falls self-efficacy and gait performance after gait and balance training in older people. J Am Geriatr Soc 2008; 56: 1154-6.

13. Cumming RG, Salkeld G, Thomas M, Szonyi G. Prospective study of the impact of fear of falling on activities of daily living, SF-36 scores, and nursing home admission. J Gerontol A Biol Sci Med Sci 2000; 55: M299-305.

14. Santos-Eggimann B, Karmaniola A, Seematter-Bagnoud L et al. The Lausanne cohort Lc65+: a population-based prospective study of the manifestations, determinants and outcomes of frailty. BMC Geriatr 2008; 8: 20.

15. Aminian K, Najafi B, Bula C, Leyvraz PF, Robert P. Spatio-temporal parameters of gait measured by an ambulatory system using miniature gyroscopes. J Biomech 2002; 35: 689-99.

16. Hausdorff JM, Rios DA, Edelberg HK. Gait variability and fall risk in community-living older adults: a 1-year prospective study. Arch Phys Med Rehabil 2001; 82: 1050-6.

17. Yardley L, Todd C, Beyer N, Hauer K, Kempen R, Piot-Ziegler C. Development and initial validation of the Falls Efficacy Scale International (FES-I). Age Ageing 2005; 34: 614-9.

18. Barnett A, Smith B, Lord SR, Williams M, Baumand A. Community-based group exercise improves balance and reduces falls in at-risk older people: a randomised controlled trial. Age Ageing 2003; 32: 407-14.

19. Zijlstra G, van Haastregt JC, van Eijk JT, Kempen GI. Evaluating an intervention to reduce fear of falling and associated activity restriction in elderly persons: design of a randomised controlled trial [ISRCTN43792817]. BMC Public Health 2005; 5: 26.

20. Perera S, Mody SH, Woodman RC, Studenski SA. Meaningful change and responsiveness in common physical performance measures in older adults. J Am Geriatr Soc 2006; 54: 743-9. 